\title{
MONDIAN DARK MATTER, ENTROPIC GRAVITY, AND INFINITE STATISTICS
}

\author{
Y. JACK NG \\ Institute of Field Physics, Department of Physics \& Astronomy \\ Chapel Hill, NC 27599-3255, USA \\ E-mail:yjng@physics.unc.edu
}

\begin{abstract}
We propose the concept of MoNDian dark matter which behaves like cold dark matter at cluster and cosmic scales but emulates modified Newtonian dynamics at the galactic scale. The connection between global physics and local galactic dynamics is implemented via entropic gravity. We also give an alternative formulation of MoNDian dark matter by using an effective gravitational Born-Infeld theory. In the latter approach, we show that the quanta of MoNDian dark matter obey infinite statistics.
\end{abstract}

Keywords: dark matter, modified Newtonian dynamics, entropic gravity, infinite statistics

\section{Introduction and Summary}

This talk is based on work ${ }^{1}$ done in collaboration with C.M. Ho and D. Minic a As the first part of the title indicates, the subject matter has to do with dark matter and modified Newtonian dynamics (MoND). Rarely does one hear these two disparate things mentioned in the same sentence. But Ho, Minic and I think that it is natural to put them together: in a real sense, they are dual to each other. $\mathrm{B}$ C

It is well-known that cold dark matter (DM) works wonders for cosmology. However, at the galactic scale, dark matter does not fare nearly as well, for (1) it can explain the observed asymptotic independence of orbital velocities on the size of the orbit only by fitting data (usually with two parameters) for individual galaxies, and (2) it cannot explain, in a natural manner, the observed baryonic Tully-Fisher (TF) relation, i.e., the asymptotic-velocity-mass $\left(v^{4} \propto M\right)$ relation.

This is in great contrast to modified Newtonian dyanmics (MoND), a scheme due to M. Milgrom. MoND stipulates that the acceleration of a test mass $m$ due to the source $M$ is given by $a=a_{N}$ for $a \gg a_{c}$, and $a=\sqrt{a_{N} a_{c}}$ for $a \ll a_{c}$, where $a_{N}=$ $G M / r^{2}$ is the usual Newtonian acceleration and the critical acceleration is given by $a_{c} \sim 10^{-8} \mathrm{~cm} / \mathrm{s}^{2}(\approx c H /(2 \pi)$ numerically, $H$ being the Hubble parameter $)$. With only one parameter (viz., $a_{c}$ ) MoND can explain fairly successfully the observed flat galactic rotation curves and the observed TF relation. But admittedly there are problems with MoND at the cluster and cosmological scales.

Thus DM and MoND complement each other well, each being successful where the other is less so. It is natural for us to combine their salient successful features into a unified scheme - all within the framework of quantum gravity ${ }^{1]}$ Significantly we

a Supported in part by the US Department of Energy.

${ }^{b}$ We note that M. Kaplinghat and M.S. Turner have attempted to derive MoND from cold dark matter, but this work has completely ignored the low-surface-brightness galaxies in which the acceleration is everywhere smaller than the critical acceleration introduced in MoND.

${ }^{\mathrm{c}}$ Proper references to work due to other authors can be found in Ref. 1. 
find that (1) MoND is a phenomenological consequence of quantum gravity, with the critical galactic acceleration $a_{c}$ correctly predicted to have magnitude $\sqrt{\Lambda / 3} \sim H$ ( $\Lambda$ denotes the cosmological constant); (2) the MoNDian force law, at the galactic scale, is merely a manifestation of DM; (3) global physics $(\Lambda)$ and local galactic dynamics $\left(a_{c}\right)$ are connected; (4) the mass profile of DM is related to the energy contents due to ordinary matter and dark energy $(\Lambda)$; $(5)$ in the formulation of MoNDian dark matter (MDM) via gravitational Born-Infeld theory, the quanta of MDM are shown to obey infinite statistics (IS), like the "particles" constituting dark energy as proposed in the holographic-quantum-foam-inspired cosmology 选.

\section{Reinterpretation of MoND via Eric Verlinde's Entropic Gravity}

Noting that Newton's law of gravity is precisely the fundamental relation that Milgrom proposes to modify so as to fit galactic rotation curves \& TF relation, we choose to start with Verlinde's recent proposal of entropic gravity (based in part on Jacobson's idea that gravity is just an effect of bodies increasing their entropy.)

First let us review how Verlinde rederives the canonical Newton's laws.

(I) Succintly Verlinde derives Newton's 2nd law $\vec{F}=m \vec{a}$, by using (a) the first law of thermodynamics to propose the concept of entropic force $F_{\text {entropic }}=T \frac{\Delta S}{\Delta x}$, and invoking Bekenstein's original arguments concerning the entropy $S$ of black holes: $\Delta S=2 \pi k_{B} \frac{m c}{\hbar} \Delta x$; and (b) the formula for the Unruh temperature, $k_{B} T=$ $\frac{\hbar a}{2 \pi c}$, associated with a uniformly accelerating (Rindler) observer.

(II). Next Verlinde rederives Newton's law of gravity $a=G M / r^{2}$ by considering an imaginary quasi-local (spherical) holographic screen of area $A=4 \pi r^{2}$ with temperature $T$, and using (a) Equipartition of energy $E=\frac{1}{2} N k_{B} T$ with $N=$ $A c^{3} /(G \hbar)$ being the total number of degrees of freedom (bits) on the screen; and (b) the Unruh temperature formula and the fact that $E=M c^{2}$.

Since we live in an accelerating universe, let us generalize Verlinde's proposal to de Sitter (dS) space with positive cosmological constant $\Lambda$. In such a dS space, the Unruh-Hawking temperature, as measured by an inertial observer, is $T_{d S}=\frac{1}{2 \pi k_{B}} a_{0}$ where $a_{0}=\sqrt{\Lambda / 3} \sim H$ (numerically). The net temperature as measured by the non-inertial observer (due to some matter sources that cause the acceleration $a$ ) is $\tilde{T} \equiv T_{d S+a}-T_{d S}=\frac{1}{2 \pi k_{B}}\left[\sqrt{a^{2}+a_{0}^{2}}-a_{0}\right]$. Part (I) of Verlinde's approach can now be generalized to yield the entropic force (in deSitter space) $F_{\text {entropic }}=\tilde{T} \nabla_{x} S=$ $m\left[\sqrt{a^{2}+a_{0}^{2}}-a_{0}\right]$. For $a \gg a_{0}$, we have $F_{\text {entropic }} \approx m a$. For $a \ll a_{0}: F_{\text {entropic }} \approx$ $m \frac{a^{2}}{2 a_{0}}$, so the terminal velocity $v$ of the test mass $m$ should be determined from $m a^{2} /\left(2 a_{0}\right)=m v^{2} / r$. In the small acceleration $a \ll a_{0}$ regime, the observed flat galactic rotation curves ( $v$ is independent of $r$ ) and the observed TF relation now require (recall $\left.a_{N}=G M / r^{2}\right)$ that $a \approx\left(2 a_{N} a_{0}^{3} / \pi\right)^{\frac{1}{4}}$. But that means $F_{\text {entropic }} \approx$ $m \frac{a^{2}}{2 a_{0}}=F_{\text {Milgrom }} \approx m \sqrt{a_{N} a_{c}}$. Thus we have recovered MoND - provided we

\footnotetext{
dThus, the quanta constituting the dark sector obey infinite statistics, rather than Fermi or Bose statistics. This may be the main difference between dark energy/matter and ordinary matter.
} 
identify $a_{0} \approx 2 \pi a_{c}$, with the critical galactic acceleration $a_{c} \sim \sqrt{\Lambda / 3} \sim H \sim$ $10^{-8} \mathrm{~cm} / \mathrm{s}^{2}$. From our perspective, MoND is a phenomenological consequence of quantum gravity; furthermore we have correctly predicted the magnitude of $a_{c}$ !

Part (II) of Verlinde's argument is straightforwardly generalized to give $2 \pi k_{B} \tilde{T}=\frac{G \tilde{M}}{r^{2}}$, where $\tilde{M}=M+M^{\prime}$ represents the total mass enclosed within the volume $V=4 \pi r^{3} / 3$, with $M^{\prime}$ being some unknown mass, i.e., dark matter. Now consistency demands that $M^{\prime}=\frac{1}{\pi}\left(\frac{a_{0}}{a}\right)^{2} M$; that is, $F_{\text {entropic }}=m\left[\sqrt{a^{2}+a_{0}^{2}}-\right.$ $\left.a_{0}\right]=m a_{N}\left[1+\left(a_{0} / a\right)^{2} / \pi\right]$. For $a \gg a_{0}$, we recover the Newtonian force law $F_{\text {entropic }} \approx m a \approx m a_{N}$, and hence $a=a_{N}$ (and $M^{\prime} \approx 0$ ). But for $a \ll a_{0}$, we have $F_{\text {entropic }} \approx m \frac{a^{2}}{2 a_{0}} \approx m a_{N}(1 / \pi)\left(a_{0} / a\right)^{2}$, yielding $a=\left(2 a_{N} a_{0}^{3} / \pi\right)^{\frac{1}{4}}$ (as required) and the dark matter mass profile $M^{\prime} \sim(\sqrt{\Lambda} / G)^{1 / 2} M^{1 / 2} r$.

The DM-MoND duality is now apparent: On one hand, we can interpret the $F_{\text {entropic }}$ equation to mean that there is no dark matter, but that the law of gravity is modified (according to MoND). On the other hand, we can rewrite $F_{\text {entropic }}$ equation as $F_{\text {entropic }}=m G\left(M+M^{\prime}\right) / r^{2}$, with $M^{\prime}$ denoting the dark matter which, by construction, is compatible with MoND. At galactic scales, $M^{\prime}, \Lambda$ and $M$ are related to one another. Dark matter of this kind can behave as if there is no dark matter but MoND. Therefore, we call it "MoNDian dark matter".

While the MoNDian dark matter profile given by $M^{\prime}=\frac{1}{\pi}\left(\frac{a_{0}}{a}\right)^{2} M$ reproduces the correct force laws (to the leading order) in both regimes of $a \gg a_{0}$ and $a \ll a_{0}$, we expect a more generic profile of the form $M^{\prime}=\left[\lambda a_{0} / a+\left(a_{0} / a\right)^{2} / \pi\right] M$, with $\lambda>0$ (and of order 1 ) which ensures that $M^{\prime}>0$ when $a \gg a_{0}$. As a function of $r$, the dark matter profile now reads (for $a \gg a_{0}$ ): $M^{\prime} \approx$ $\left[\lambda\left(a_{0} / a_{N}\right)+\left(\frac{1}{\pi}-\lambda(1+\lambda)\right)\left(a_{0} / a_{N}\right)^{2}\right] M$. In principle, this mass profile can be checked against observational data.

We conclude this section with an observation on the application of MoNDian dark matter to cosmology 1 One can in principle have Einstein's gravity together with a MoNDian dark matter source. The departure from MoND happens when we replace $\tilde{M}$ with the active gravitational (Tolman-Komar) mass, i.e. when a nonrelativistic source is replaced by a fully relativistic source. In that case, we have $\sqrt{a^{2}+a_{0}^{2}}-a_{0}=\frac{G\left(M(t)+M^{\prime}(t)\right)}{\tilde{r}^{2}}+4 \pi G p \tilde{r}-\frac{\Lambda}{3} \tilde{r}$, where $\tilde{r}$ is the physical radius. If we naively use MoND at the cluster scale, we would be missing the pressure $(p)$ and cosmological constant terms $4 \pi G p \tilde{r}-\frac{\Lambda}{3} \tilde{r}$ which could be significant. This may explain why MoND doesn't work well at the cluster scale, despite the DM-MoND duality realized at the galactic scale.

\section{MoNDian Dark Matter via Gravitational Born-Infeld Theory}

A particularly useful reformulation of MoND is via an effective gravitational dielectric medium, motivated by the analogy between Coulomb's law in a dielectric 
medium and Milgrom's law for MoND. $\mathrm{e}$ Starting from the Born-Infeld theory of electrostatics, we can write the corresponding gravitational Hamiltonian density as $H_{g}=b^{2}\left(\sqrt{1+D_{g}^{2} / b^{2}}-1\right) /(4 \pi)$, where $D$ stands for the displacement vector and $b$ is the maximum field strength in the Born-Infeld theory. With $A_{0} \equiv b^{2}$ and $\vec{A} \equiv b \overrightarrow{D_{g}}$, the Hamiltonian density becomes $H_{g}=\left(\sqrt{A^{2}+A_{0}^{2}}-A_{0}\right) /(4 \pi)$.

As in the Verlinde approach, let us assume energy equipartition. Then the effective gravitational Hamiltonian density is equal to $H_{g}=\frac{1}{2} k_{B} T_{\text {eff }}$. The Unruh temperature formula $T_{e f f}=\frac{\hbar}{2 \pi k_{B}} a_{\text {eff }}$ implies that the effective acceleration is given by $a_{\text {eff }}=\sqrt{A^{2}+A_{0}^{2}}-A_{0}$. Next we make use of the equivalence principle which suggests that we should identify (at least locally) the local accelerations $\vec{a}$ and $\vec{a}_{0}$ with the local gravitational fields $\vec{A}$ and $\vec{A}_{0}$ respectively, viz., $\vec{a} \equiv \vec{A}, \quad \vec{a}_{0} \equiv \vec{A}_{0}$. Then $a_{\text {eff }}$ should be identified as $a_{\text {eff }} \equiv \sqrt{a^{2}+a_{0}^{2}}-a_{0}$, which, in turn, implies that the Born-Infeld inspired force law takes the form (for a given test mass $m$ ) $F_{\mathrm{BI}}=m\left(\sqrt{a^{2}+a_{0}^{2}}-a_{0}\right)$, which is precisely the MoNDian force law!

To be a viable cold dark matter candidate, the quanta of our MoNDian dark

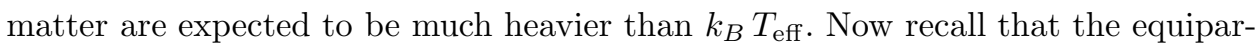
tition theorem in general states that the average of the Hamiltonian is given by $\langle H\rangle=-\frac{\partial \log Z(\beta)}{\partial \beta}$, where $\beta^{-1}=k_{B} T$ and $Z$ denotes the partition function. To obtain $\langle H\rangle=\frac{1}{2} k_{B} T$ per degree of freedom, even for very low temperature, we require $Z$ to be of the Boltzmann form $Z=\exp (-\beta H)$. $\mathrm{f}$ But this is precisely what is called the infinite statistics $g$ as described by the Cuntz algebra (a curious average of the bosonic and fermionic algebras) $a_{i} a_{j}^{\dagger}=\delta_{i j}$. It is intriguing that only by invoking infinite statistics for the microscopic quanta which underly the thermodynamic description of gravity implying such a MoNDian force law, can the assumption of energy equipartition, even for very low temperature $T_{\text {eff }}$, be justified.

\section{References}

1. C. M. Ho, D. Minic and Y. J. Ng, Phys. Lett. B 693, 567 (2010); Gen. Rel. Grav. 43, 2567 (2011) (arXiv:1105.2916); Phys. Rev. D 85, 104033 (2012) (arXiv:1201.2365).

2. Y. J. Ng, in Proc. of the 12th Marcel Grossmann Meeting on General Relativity, ed. by T. Damour et al. (World Scientific, Singapore, 2012), 2435 (arXiv:1001.0411); in Proc. of the 3rd International Conference on Time and Matter, ed. by M. O'Loughin et al. (University of Nova Gorica Press, Slovenia, 2011), 103 (arXiv:1102.4109). Also see V. Jejjala, M. Kavic and D. Minic, Adv. High Energy Phys. 2007, 21586 (2007).

\footnotetext{
e It has been known to L. Blanchet, Milgrom and others that the MoNDian force law can be formulated as being governed by a nonlinear generalization of Poisson's equation which describes the nonlinear electrostatics embodied in the Born-Infeld theory.

${ }^{f}$ Note that the conventional quantum-mechanical Bose-Einstein or Fermi-Dirac statistics would not lead to $\langle H\rangle=\frac{1}{2} k_{B} T$ per degree of freedom at low temperataure.

gNotable properties of infinite statistics include: particles obeying IS are virtually distinguishable; the partition function is $Z=\Sigma e^{-\beta H}$, with NO Gibbs factor; in IS, all representations of the particle permutation group can occur; theories of particles obeying IS are non-local; TCP theorem and cluster decomposition still hold; and quantum field theories with IS remain unitary.
} 